\title{
THE PHOTOGRAPHIC STUDY OF IMPACT AT MINIMAL VELOCITIES.
}

By C. V. RAMAN.

\section{INTRODUCTION.}

UERTZ'S well-known theory ${ }^{1}$ treats the phenomena occurring during elastic impact practically as statical effects; the local compression at the place of contact is regarded as gradually produced and subsiding completely by reversal of the process by which it is produced. In the particular case of two solid spheres impinging on each other, the theory finds its own justification by showing that the duration of impact is large compared with the gravest period of vibration of either body. The results indicated by Hertz's theory for the duration of impact, for the radius of the area of contact, and for the approach of the centers of the spheres have been compared with those found in experiment by various investigators among whom may be mentioned Schneebeli, Hamburger, ${ }^{2}$ and more recently, Lafay, ${ }^{3}$ Kennelly and Northrup. ${ }^{4}$ Hertz's theory is incomplete in that it entirely ignores the dissipation of energy that occurs during collision. Recently, however, Lord Rayleigh ${ }^{5}$ has extended Hertz's work by investigating the circumstances of the first appearance of vibrations in the case of two impinging spheres, and has shown that the proportion of translational energy transformed into energy of vibration is negligibly small over a considerable range of velocities. The dissipation of energy that occurs in the impact of spheres has thus to be assigned principally to two causes, $(a)$ the stresses occurring during impact exceeding the limits within which elastic recovery is immediate and perfect, and $(b)$ the production of sound. The second of these causes has been recently investigated at Calcutta by Banerji, ${ }^{6}$ who has shown that the sound waves produced by the impact of spheres are practically

1 Hertz's Miscellaneous Papers, English edition, p. I46, and Love's Treatise on Elasticity, 2d edition, p. I95.

2 Schneebeli, Rep. d. Phys., Bd. 22, I886, and Hamburger, Tageblatt d. Nat. Vers. in Wiesbaden, 1887 .

${ }^{3}$ Lafay, Comptes Rendus, p. 525, I900, and Anna. Chim. Phys., p. 24I, I90I.

4 Kennelly and Northrup, Frank. Inst. Journ., July, Igrr.

5 Rayleigh, Phil. Mag., Vol. XI., p. 283, 1906.

6 Banerji, Phil. Mag., July, I9r6, and January, I918. 
due to the impulse communicated to the atmosphere by the sudden reversal of the motion of the spheres. The energy thus lost may be evaluated mathematically, and being directly proportional to the density of the atmosphere, can be made as small as desired by sufficiently reducing the pressure. If we leave it out of consideration, the dissipation of energy has to be ascribed entirely to failure of perfect elasticity during impact. According to Hertz, if two steel spheres $2.5 \mathrm{~cm}$. radius meet with a relative velocity of only $\mathrm{I} \mathrm{cm}$. per second, the maximum pressure developed during impact is 7,300 kilogrammes per sq. $\mathrm{cm}$. Stresses of this order of magnitude would, in the case of most materials overstep the elastic limits and result in dissipation of energy. If the substance of the spheres be plastic, the energy of collision may even be dissipated entirely in the production of deformation. This is illustrated by some recent interesting experiments by Pochettino ${ }^{1}$ who studied the elasticity, the viscosity, and the coefficient of restitution of pitch at different temperatures. The coefficient of restitution was 0.92 at $10^{\circ} \mathrm{C}$., fell to 0.44 at $28 . \mathrm{I}^{\circ} \mathrm{C}$., to 0.12 at $3 \mathrm{I}^{\circ} \mathrm{C}$, , and was zero at $32^{\circ} \mathrm{C}$.

The question naturally arises whether the coefficient of restitution can be connected in some definite manner with the magnitude of the stresses produced by impact. According to Hertz's theory, the maximum pressure developed per unit area is proportional to the two-fifths power of the velocity before impact, and is independent of the size of the balls. Accordingly, by reducing the velocity of impact sufficiently, it should be possible to keep the stresses developed below any definite value which may be assigned as the elastic limit, and the coefficient of restitution for such velocities should approach unity for all solids. That the coefficient becomes smaller with increasing velocity of impact has indeed been shown by Hodgkinson, ${ }^{2}$ and more recently also by Vincent. ${ }^{3}$ In view, however, of the bearing of the subject on the theory of impact, it appeared worth while to make a more detailed examination, and to push the observations to the smallest velocities up to which it is possible to obtain regular results. In order to make reliable measurements at such small velocities, it was decided to use the photographic method to record the relative motions of the spheres immediately before and after impact, and to determine the coefficient of restitution at leisure by measurements of the plates. The paper presents the first instalment of the results of the work in the form of graphs (Figs. I to 5) showing the relation between the coefficient of restitution and the velocity of impact for polished spheres

1 Pochettino, N. Cimento, Aug, I9r4.

${ }^{2}$ Hodgkinson, Report Britt. Assoc., I834.

3 Vincent, Camb. Phil. Soc., Proc., August, I9oo. 
of equal radius of (I) brass, (2) aluminium, (3) hard bronze, (4) white marble and (5) lead.

\section{Experimental Methods and Results.}

The impinging spheres were hung by bifilar suspensions, and were held apart and dropped electro-magnetically by the aid of small iron washers

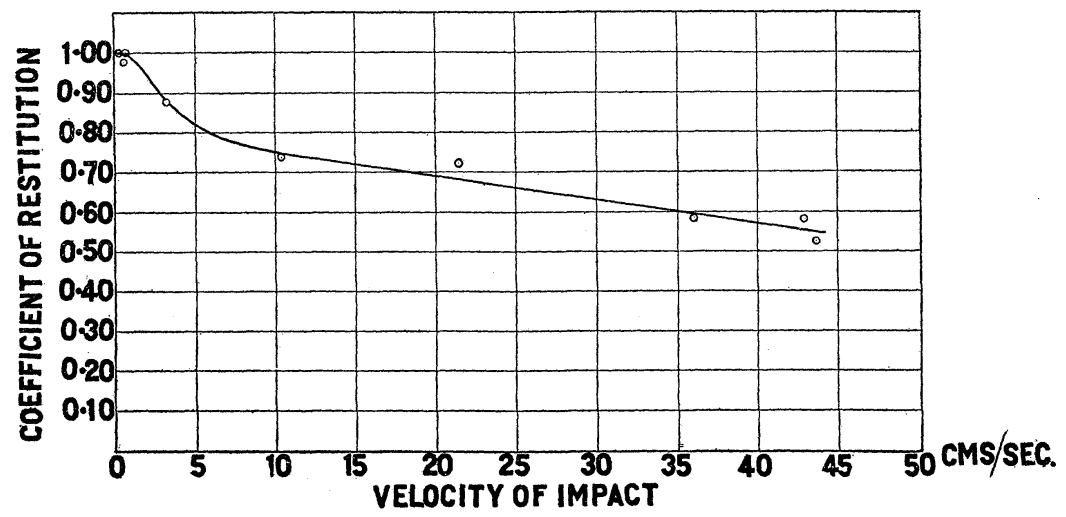

Fig. 1.

Brass spheres.

firmly fixed near the point of suspension. The motion of the balls before and after impact was recorded photographically in the following manner. A fine horizontal slit was illuminated by an electric arc, and an image of

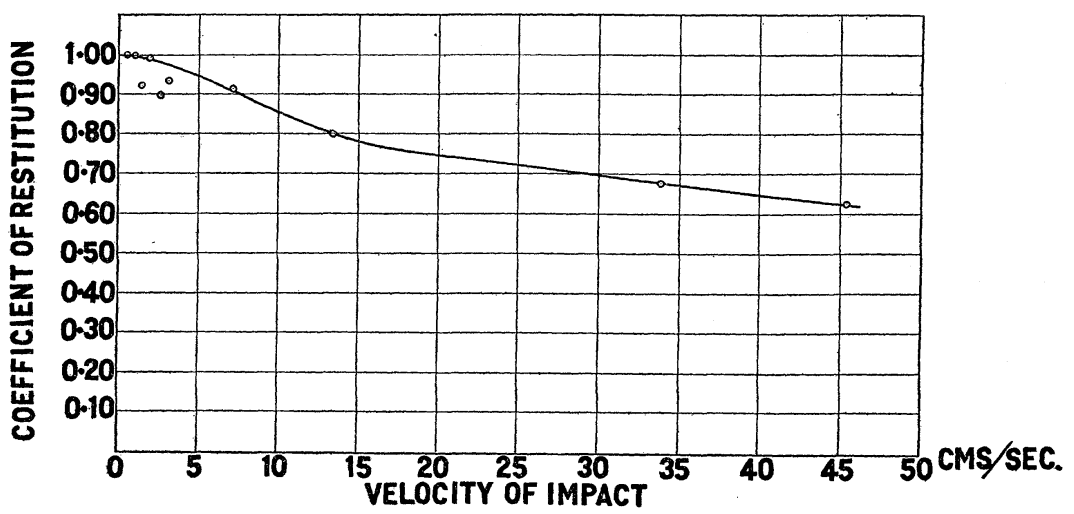

Fig. 2.

Aluminum Spheres.

it was focused on the lowest point of the circle on which the centers of the balls move when dropped, and so adjusted that when the balls hang 
in the position of equilibrium, the image of the slit is just completely obscured, and appears only when the balls are drawn apart. A second lens refocuses the image of the slit seen between the balls, on a photographic plate which is caused to move vertically downwards when the balls are released. A fairly uniform vertical motion of the plate was obtained by

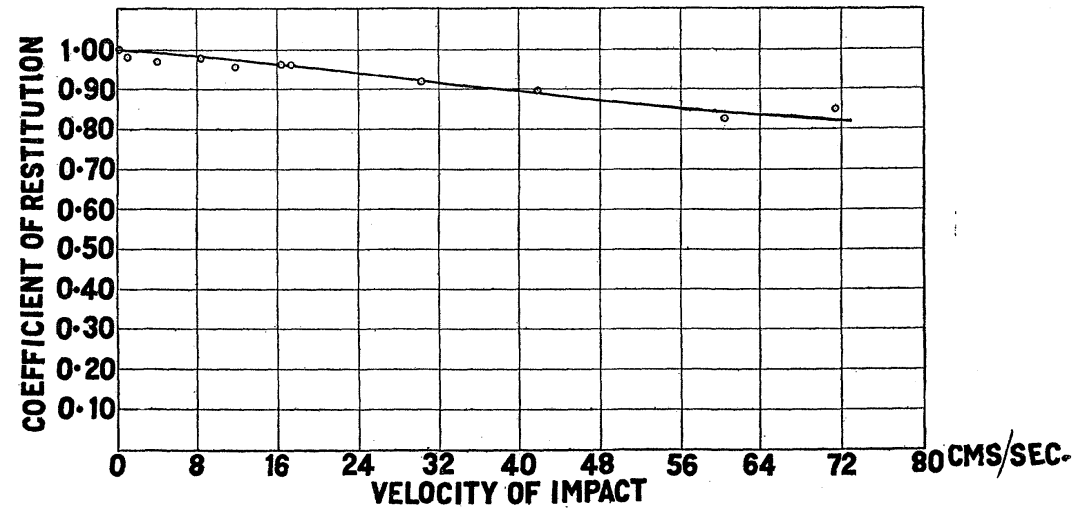

Fig. 3.

Hard Bronze Spheres.

suspending the dark slide from a string wrapped round the axle of a small fly wheel, and setting the latter in rotation. The dark slide moves down in vertical grooves at the back of the camera which is completely covered

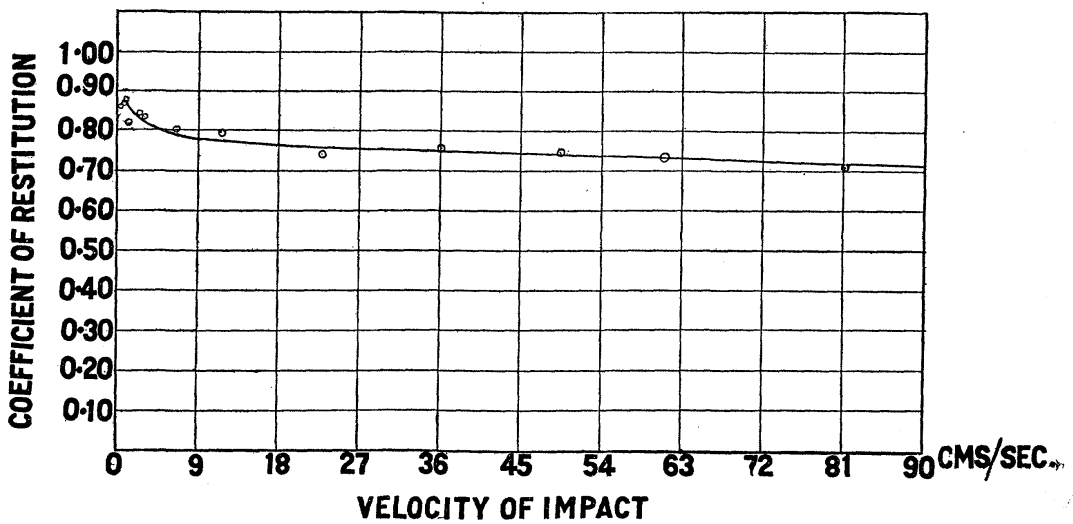

Fig. 4.

Marble spheres.

up with the exception of a small slit through which the light falls on the plate. With this arrangement the photograph can be taken without any need for specially darkening the room. The vibration of a tuning: 
fork of frequency 60 d.v. is simultaneously recorded on the photographic plate, and serves both as a check on the approximate uniformity of the motion and also as a scale of time against which the relative velocity of the balls is measured. A specimen photograph is reproduced as Fig. 6, see accompanying plate. The width of the wedges of light that appear immediately before and after the impact is measured at two or three points on each side, and gives directly the relative motion of the balls during the corresponding intervals of time. The ratio gives the coefficient of restitution. The magnification of linear dimensions on the plate is measured once for all by photographing a scale held in the plane containing the centers of the impinging spheres. By using long suspensions (2,

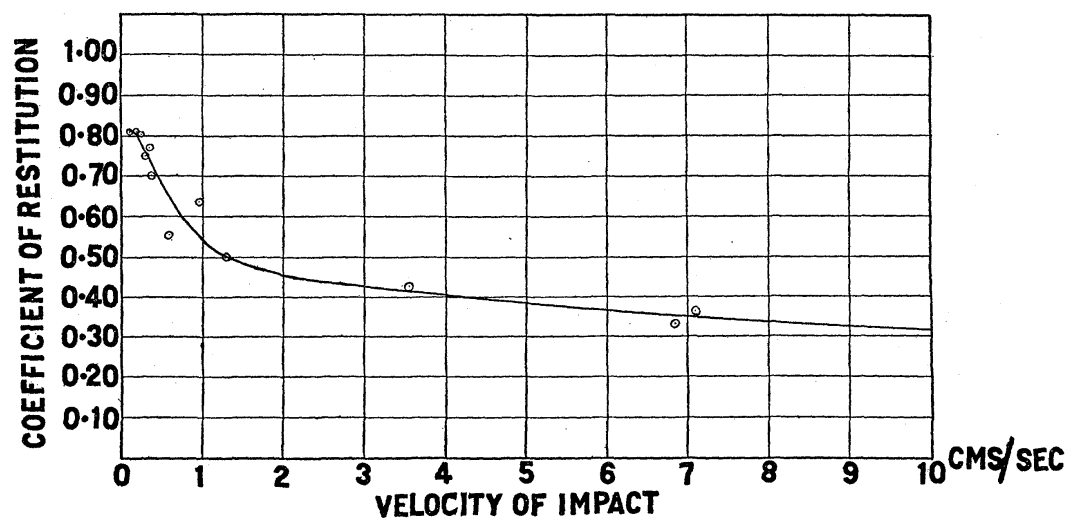

Fig. 5.

Lead spheres.

and sometimes 5 meters length), very small velocities can be obtained without an undue diminution in the amplitude of motion of the balls. ${ }^{1}$

The method was found very simple and convenient in practice, as the record once secured could be studied and measured at leisure. The most rapid way of measuring the photographs was to project them on a screen with a lantern, and to read off the widths of the wedges with an ordinary millimeter scale. An alternative which, however, proved much less convenient was to measure the photographs on a cross-slide micrometer.

It was found impossible to obtain regular and consistent results at low velocities of impact unless the surfaces of the balls were clean and highly polished. The choice of materials for the work was somewhat restricted

1 In the course of the work, the curious observation was made that the suspensions of the spheres were set in oscillation as the result of impact in a mode which is initially the same as that of a bowed string observed by Helmholicz. (See the paper on Discontinuous Wave-motion by Raman and Appasamaiyar, Phil. Mag., January, 1916.) 
Physical Review, Vol. Xil., Second Series. December, I9I8.
Plate 1.

To face page 446 .

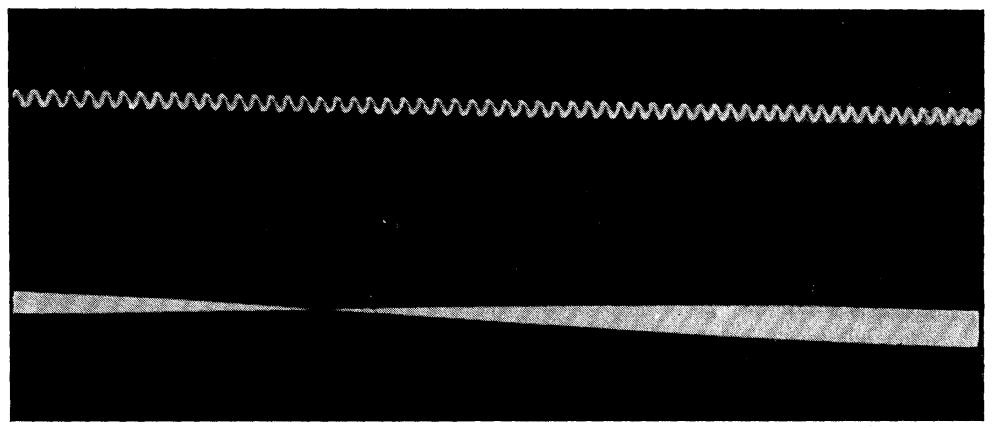

FIG. 6.

C. V. RAMAN. 
by this consideration. According to recent views, polishing results in the formation of a surface film whose elastic properties are somewhat different from those of the bulk of the substance, and the complication introduced by this fact though undesirable, could not be avoided. Nevertheless the results indicated in Figs. I to 5 are sufficient to show that the coefficient of restitution makes a decided approach towa ds unity for a very small velocity of impact. The most noteworthy case is that of lead, in which this rise is particularly rapid. With marble spheres, it was found difficult to obtain a thoroughly satisfactory polish to begin with, and the breaking up of the crystalline structure at the place of contact as the result of impact was very evident to inspection. It was not therefore surprising that in the case of marble somewhat irregular results were obtained when it was attempted to push the observations down to very low velocities of impact. It is hoped when a suitable opportunity arises to continue this investigation in various directions, particularly as regards $(a)$ the results obtained when the impinging spheres are of unequal diameters or of different materials; $(b)$ to determine in what manner the apparent coefficient of restitution is affected by the density of the surrounding fluid, and $(c)$ to give a fuller theoretical discussion of the results.

2Io, BowbazaAR Street,

Calcutta, INDia,

May I4, I9I8. 


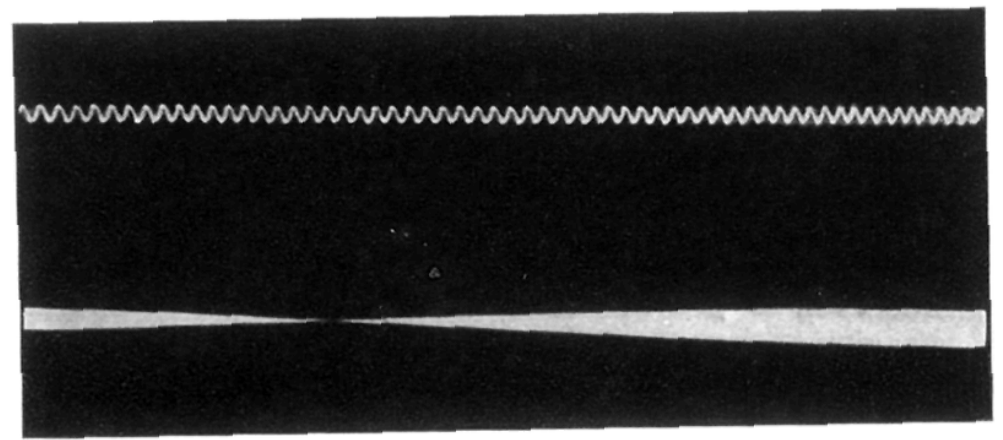

FIG. 6. 